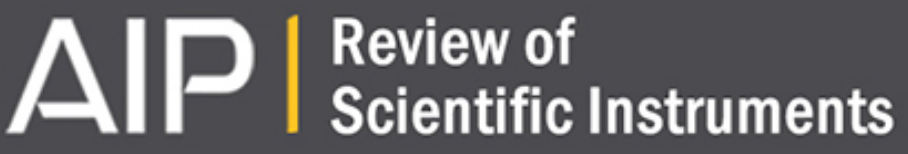

High-speed high-resolution plasma spectroscopy using spatial-multiplex coherence imaging techniques (invited)

John Howard

Citation: Review of Scientific Instruments 77, 10F111 (2006); doi: 10.1063/1.2219433

View online: http://dx.doi.org/10.1063/1.2219433

View Table of Contents: http://scitation.aip.org/content/aip/journal/rsi/77/10?ver=pdfcov

Published by the AIP Publishing

The Complete Guide to Building an Instrument Control System

» Learn more at www.NI.com/Instrument-Control

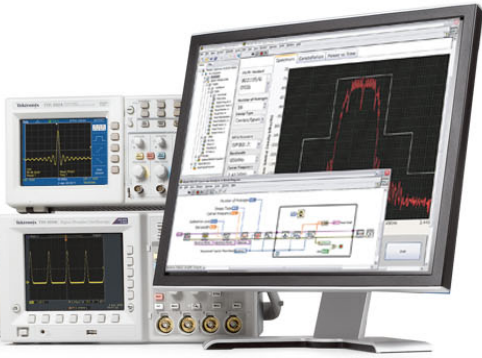




\title{
High-speed high-resolution plasma spectroscopy using spatial-multiplex coherence imaging techniques (invited)
}

\author{
John Howard ${ }^{\text {a) }}$ \\ Plasma Research Laboratory, Australian National University, Canberra ACT 0200, Australia
}

(Received 8 May 2006; presented on 10 May 2006; accepted 7 June 2006; published online 29 September 2006)

\begin{abstract}
We have recently obtained simultaneous two-dimensional (2D) plasma Doppler spectroscopic images of plasma brightness, temperature, and flow fields. Using compact polarization optical methods, quadrature images of the optical coherence of an isolated spectral line are multiplexed to four quadrants of a fast charge-coupled device camera. The simultaneously captured, but distinct, images can be simply processed to unfold the plasma brightness, temperature, and flow fields. This static system, which is a spatial-multiplex variant of previously reported electro-optically modulated, temporal-multiplex coherence imaging systems, is based on a high-throughput imaging polarization interferometer that employs crossed Wollaston prisms and appropriate image plane masks. Because the images are captured simultaneously, it is well suited to high-spectral-resolution, high-throughput 2D imaging of transient or rapidly changing spectroscopic scenes. To illustrate instrument performance we present recent results using a static 4-quadrant Doppler coherence imaging on the $\mathrm{H}-1$ heliac at the ANU. (C) 2006 American Institute of Physics.
\end{abstract}

[DOI: 10.1063/1.2219433]

\section{INTRODUCTION}

In recent years we have been developing electrooptically modulated polarization interferometers for widefield time-resolved "coherence imaging," with applications in plasma Doppler spectroscopy, polarization spectroscopy, relative line intensity measurements, laser Thomson scattering, and infrared thermography. ${ }^{1-3}$ When the spectral content of a scene can be successfully represented by a small number of free parameters (e.g., motional Stark effect, Zeeman effect, thermography, bremsstrahlung, Thomson scattering), it is often the case that these parameters can be recovered from measurements of the complex coherence (phase and amplitude of the interferogram) at a small number of appropriately chosen fixed optical path length delays. Because there is no entrance slit, the use of interferometric methods provides high light throughput and opens the possibility of twodimensional high-resolution imaging.

In the case of a Doppler broadened multiplet, the fringe visibility at an optical delay comparable with the spectral line coherence length will decrease as the species temperature increases. This change can be measured by dithering or stepping the optical path length in order to characterize the local fringe pattern. If the line center frequency is Doppler shifted, it is also possible to obtain the associated change in the interference fringe phase. In the Doppler case, the unknown parameters are the spectral line brightness, width, and center frequency and the associated coherence quantities are the interferogram brightness, fringe contrast, and phase.

The simplest approach to acquire spectrally resolved images is to use temporal or temporal-frequency domain mul-

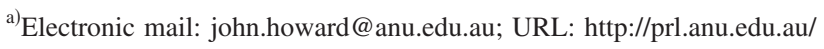

tiplex techniques. In the latter case, having first isolated the spectral feature of interest using an interference prefilter, an electro-optically path-modulated polarization interferometer allows the targeted coherence information to be encoded over a spread of harmonics of the modulation frequency. The modulated light is typically detected using multianode phototube arrays with modulation frequencies of $50 \mathrm{kHz}$ and path length amplitudes up to half a wave. Temporal multiplex methods are also well suited to charge-coupled device (CCD) cameras where the interferometer path difference can be electro-optically stepped synchronously with the camera frame rate. ${ }^{4}$ In this case, however, temporal resolution is limited by the camera frame rate and the required number of path length steps (minimum of 3 ).

An alternative completely static approach, the subject of this article, utilizes a pair of Wollaston prisms to produce independent images of the optical coherence in four quadrants of the CCD array. Because the distinct coherence images are sampled simultaneously, the static arrangement allows the capture and spectral imaging analysis of fast transient plasma events. Combined with field-of-view widening techniques, polarization manipulation, and suitable imaging optics, it is possible to perform fast, high-throughput, time-resolved two-dimensional (2D) spectral imaging.

The article is organized as follows. Section 2 briefly reviews the operating principles of spectroscopic interferometry with emphasis on application to Doppler tomographic measurements of temperature and flow. This is followed by a description of time domain modulation techniques applied to Doppler coherence imaging with some example data from the H-1 heliac at the Australian National University. A static version of the instrument is then presented in Sec. IV along with plasma results. 


\section{COHERENCE METHODS FOR DOPPLER SPECTROSCOPY AND TOMOGRAPHY}

The Fourier transform spectrometer (interferometer) splits an incident scalar wave component, relatively delaying the nominally equal amplitude components by time $\tau$ before they are recombined at a square-law detector. For quasimonochromatic light of center frequency $\nu_{0}$, the signals at the complementary interferometer output ports are proportional to $^{3}$

$$
S_{ \pm}\left(\phi_{0}\right)=\frac{I_{0}}{2}\left[1 \pm \Re\left[\gamma\left(\kappa \phi_{0}\right) \exp \left(i \phi_{0}\right)\right]\right],
$$

where $I_{0}$ is the spectral line brightness, $\phi_{0}=2 \pi \nu_{0} \tau_{0}$, where $\tau_{0}$ is the center-frequency time delay, and the factor

$$
\kappa=1+\left.\frac{\nu_{0}}{\tau_{0}} \frac{\partial \tau}{\partial \nu}\right|_{\nu_{0}}
$$

accounts for any optical frequency dispersion of the time delay. The self-coherence of the spectral line $\gamma$ is given by

$$
\gamma(\phi)=\frac{1}{I_{0}} \int_{-\infty}^{\infty} I(\xi) \exp (i \phi \xi) d \xi,
$$

where $\xi=\left(\nu-\nu_{0}\right) / \nu_{0}$ is a normalized frequency difference coordinate.

Despite the simple linear mapping from velocity distribution to emission spectrum via $\xi \equiv \boldsymbol{v} \cdot \hat{\boldsymbol{l}} / c$, interpretation of Doppler spectra is complicated by the fact that for an inhomogeneous plasma, the spectrum is the summation along the line of sight $\boldsymbol{l} \equiv|\boldsymbol{l}| \hat{\boldsymbol{l}}$ of local-brightness-weighted emission spectra of varying shape. Nevertheless, for a velocity distribution function $f\left(\boldsymbol{r}, \boldsymbol{v}-\boldsymbol{v}_{D}\right)$ that is isotropic in the frame drifting with local velocity $\boldsymbol{v}_{D}(\boldsymbol{r})$, it can be shown that multiview measurements of the light coherence at a single fixed delay will suffice for the reconstruction of the emissivity (dc), flow vorticity (phase), and species temperature (fringe visibility). ${ }^{5}$ In the drifting isotropic case, the local spectral coherence at position $\boldsymbol{r}$ in the plasma takes the simple separable form

$$
G\left(\boldsymbol{r}, \hat{\phi}_{0}\right)=\exp \left(i \hat{\phi}_{0} \boldsymbol{v}_{D} \cdot \hat{\boldsymbol{l}}\right) G_{0}\left(\boldsymbol{r}, \hat{\phi}_{0}\right),
$$

where it is convenient to introduce the group delay coordinate

$$
\hat{\phi}_{0} \equiv \kappa \phi_{0} .
$$

For the special case of a plasma drifting in local thermal equilibrium, the Fourier transform of the spectral line shape (the projected distribution function) in the locally drifting frame is given by

$$
G_{0}\left(\boldsymbol{r}, \hat{\boldsymbol{\phi}}_{0}\right)=\exp \left(-\hat{\phi}_{0}^{2} \xi_{\mathrm{th}}^{2} / 4\right) \equiv \exp \left[-T_{S}(\boldsymbol{r}) / T_{C}\right],
$$

where $T_{S}(\boldsymbol{r})$ is the local species temperature and $T_{C}$ is the instrument "characteristic temperature,"

$$
k T_{C}=\frac{1}{2} m_{S} V_{C}^{2}, \quad V_{C}=\frac{2 c}{\hat{\phi}_{0}} .
$$

For optimum sensitivity to temperature changes, the characteristic temperature is chosen to be close to the expected source temperature requiring $\hat{\phi}_{0} v_{\text {th }}=2$.

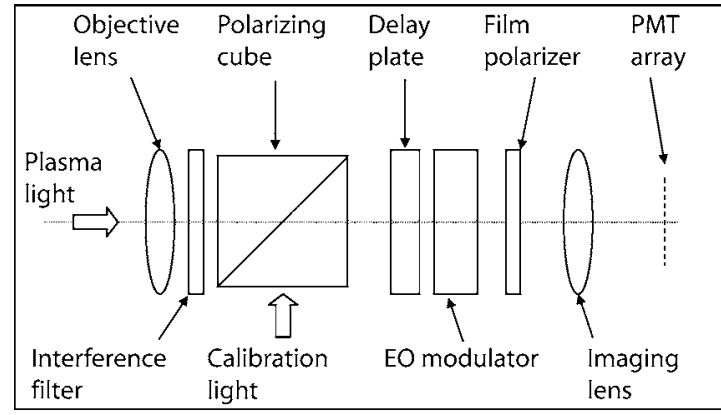

FIG. 1. Optical layout for simple single fixed delay imaging modulated polarization interferometer.

Measurement of the coherence $\gamma$ of the emission spectrum in direction $\hat{l}$ at phase delay $\phi_{0}$ delivers the fringe contrast

$$
\zeta \equiv\left|\gamma\left(\hat{\phi}_{0} ; \hat{l}\right)\right|=\frac{1}{e_{0}} \int_{L} I_{0}(\boldsymbol{r}) \exp \left[\frac{-T_{S}(\boldsymbol{r})}{T_{C}}\right] d l,
$$

where

$$
e_{0}=\int_{L} I_{0}(\boldsymbol{r}) d l
$$

is the line-integrated brightness. Note that the fringe contrast is insensitive to the drift velocity. Provided that $\hat{\phi}_{0} \boldsymbol{v}_{D} \ll 1$, a quantity related to the species flow velocity can be obtained from the interferometric phase shift

$$
\phi_{D}=\hat{\phi}_{0} \frac{1}{e_{0}} \int_{L} I_{0}(\boldsymbol{r}) \exp \left[\frac{-T_{S}(\boldsymbol{r})}{T_{C}}\right] \boldsymbol{v}_{D} \cdot d \boldsymbol{l} .
$$

The phase offset $\hat{\phi}_{0}$ can be chosen to magnify the usually small Doppler shift component, but not so large as to invalidate the first order approximation leading to Eq. (10). The important result is that measurements of fringe contrast and phase at a single optical delay can be related directly to line integral measurements of the scalar field $I_{0} G_{0}$ and longitudinal line integral measurements of the vector field $I_{0} G_{0} \boldsymbol{v}_{D}$.

\section{COHERENCE IMAGING USING TIME DOMAIN MULTIPLEX METHODS}

It is possible to use one or more electro-optically modulatable birefringent plates placed between a polarizer and analyzer to encode coherence information over a spread of harmonics of the modulation frequency. ${ }^{1}$ In the simplest case (see Fig. 1), a single fixed-delay wave plate is placed with its fast axis at $45^{\circ}$ with respect to the orientation of the first polarizer and final analyzer. The plate mutually delays the polarization components parallel and perpendicular to the fast axis. Writing $k_{0}=2 \pi \nu_{0} / c$, a plate of thickness $L$ introduces a fixed phase delay $\phi \equiv \phi_{0}$ given by

$$
\phi_{0}=k_{0} B L=2 \pi \nu_{0} \tau_{0} \equiv 2 \pi N,
$$

where $N$ is the order of interference and $B=n_{E}-n_{O}$ is the birefringence of the delay plate, where $n_{E}$ and $n_{O}$ are the extraordinary and ordinary wave refractive indices, respectively. If the wave plate is also electro-optic (e.g., $\mathrm{LiNbO}_{3}$ ), the application of a high voltage along an appropriate crystal 
axis of the wave plate will allow the optical path delay to be modulated in order to measure the local fringe properties. A modulated polarization interferometer is not subject to the mechanical and acoustic noise problems normally associated with optical Fourier transform spectrometers. This simple instrument has been called the modulated solid spectrometer or MOSS.

The simplest way to measure the coherence at delay $\phi_{0}$ is to sinusoidally modulate the optical path delay by $\phi_{1}$ $z \pi / 2$ so that the total time-varying interferometric phase is given by

$$
\widetilde{\phi}_{0}=\phi_{0}+\phi_{1} \sin \Omega t \text {. }
$$

The high voltage modulation of the electro-optic plate(s) is typically achieved using a function generator, a standard audio amplifier, and step-up transformer (100:1) to produce drive voltages of up to $4 \mathrm{kV} \mathrm{p}$.-p. at frequencies in the range of $1-50 \mathrm{kHz}$. In practice, the modulation and delay functions are often separated by using a stand-alone lithium tantalate, zero-net-delay modulator.

We have constructed a number of one-dimensional (1D) and 2D imaging systems for plasma cross-sectional imaging and tomography, using both linear and 2D multianode phototube arrays and fast framing CCD cameras. ${ }^{4,6,7}$ The cameras use standard F-mount lenses and $50 \mathrm{~mm}$ optics with typically $\sim 40 \mathrm{~mm}$ clear aperture to match that of the birefringent plates. The systems are modular in construction, using interchangeable flanged components that can be bolted to an optical rail. When the interferometer is coupled to a CCD camera, a three-step modulation scheme applied synchronously with the camera frame rate is used instead of the sinusoidal modulation.

Spectral resolution is proportional to the optical phase delay introduced by the birefringent plate. For example, a spectral line of width $0.1 \mathrm{~nm}$ and centered at $500 \mathrm{~nm}$ requires a plate producing a delay of roughly $\kappa N \sim 5000$ waves in order to be sensitive to changes in the optical coherence length. Given that the resolution of a grating spectrometer is inversely proportional to the entrance and exit slit widths, the light throughput advantage is most evident in high-resolution applications.

Contrast degradation with increasing collection solid angle can be remedied using crossed birefringent plates with an intervening half wave plate. ${ }^{8}$ The resulting field of view is expanded by a factor of $[2 \sqrt{2} n / B]^{1 / 2} \sim 8$ to typically tens of degrees or more. The instrument response can be measured by illuminating the field of view with a suitably diffused monochromatic light source (laser beam or spectral lamp) having a wavelength at or near the wavelength of interest. Issues related to characterization of the instrument function and performance are presented in detail elsewhere. ${ }^{2}$

\section{A. Doppler spectroscopy results obtained using time-multiplex systems}

A number of modulated coherence imaging systems have been installed and operated on the H-1 heliac (Refs. 6 and 7) at the RFX reversed field pinch in Padova, Italy and at the WEGA stellarator at the Max Planck Institute for Plasma Physics in Greifswald, Germany. ${ }^{4}$ A dual temporal/spatial

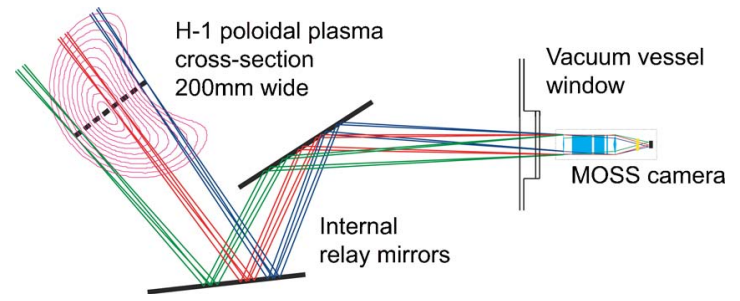

FIG. 2. Diagram showing the plasma region viewed by the H-1 MOSS camera. Mirrors internal to the vacuum vessel direct the plasma emission from a poloidal cross section to the camera. The central and extreme viewing chords are shown.

multiplex coherence imaging system has also been constructed recently for operation on the KSTAR tokamak at the Korean National Fusion Research Center.

The H-1 heliac is a coil-in-tank helical-axis toroidal magnetic plasma confinement device having a major radius $R=1 \mathrm{~m}$ and a bean-shaped plasma cross section of average minor radius $a \sim 0.2 \mathrm{~m}$. The H-1 MOSS camera is mounted in front of a vacuum tank port and views the plasma via a pair of elongated flat mirrors that are supported inside the vacuum tank (see Fig. 2). The plasma poloidal crosssectional slice is imaged through the polarization interferometer and $f / 4$ grating spectrograph prefilter onto a 16 channel multianode phototube array.

The camera has been used to study the evolution of the ion distribution function and dynamical radial force balance in rf-sustained ( $7 \mathrm{MHz}, 80 \mathrm{~kW})$ discharges in argon at low magnetic field strengths $(0.1 \mathrm{~T})$. Low field results are based on Doppler broadening of the $\mathrm{Ar} \mathrm{II}^{2} D_{5 / 2} \rightarrow{ }^{2} P_{3 / 2}$ transition at $488 \mathrm{~nm}$. Ion temperatures range between 5 and $20 \mathrm{eV}$, increasing with magnetic field strength and decreasing with neutral fill pressure. Raw projection data for a low field discharge exhibiting a spontaneous transition to improved confinement are presented in Fig. 3. At the transition the temperature increases from 9 to $12 \mathrm{eV}$ and the density profile becomes more peaked, while there is little change in the plasma flow speed, which has a maximum around $1000 \mathrm{~m} / \mathrm{s}$ and reverses about the plasma center. A more comprehensive analysis of measurements made at multiple optical delays, including measurements of the neutral velocity distribution function, indicates a strong coupling between the ion and neutral fluids and an accompanying distortion of the velocity distribution function that is well explained by kinetic modeling. ${ }^{9}$

\section{STATIC COHERENCE IMAGING SYSTEMS}

To enable single snapshot 2D spectral imaging, we have devised a polarization interferometer that multiplexes independent interferograms to four quadrants of a CCD array. In this system a Wollaston prism is used as the first polarizing element. It produces orthogonally polarized images that can thereafter be angularly multiplexed through separate, effectively independent, interferometers. A balanced separation requires that the incident light be essentially unpolarized. If this is not the case, balance can be ensured by inserting a linear polarizer oriented at $45^{\circ}$ with respect to the Wollaston splitting plane. A lens following this first Wollaston produces 

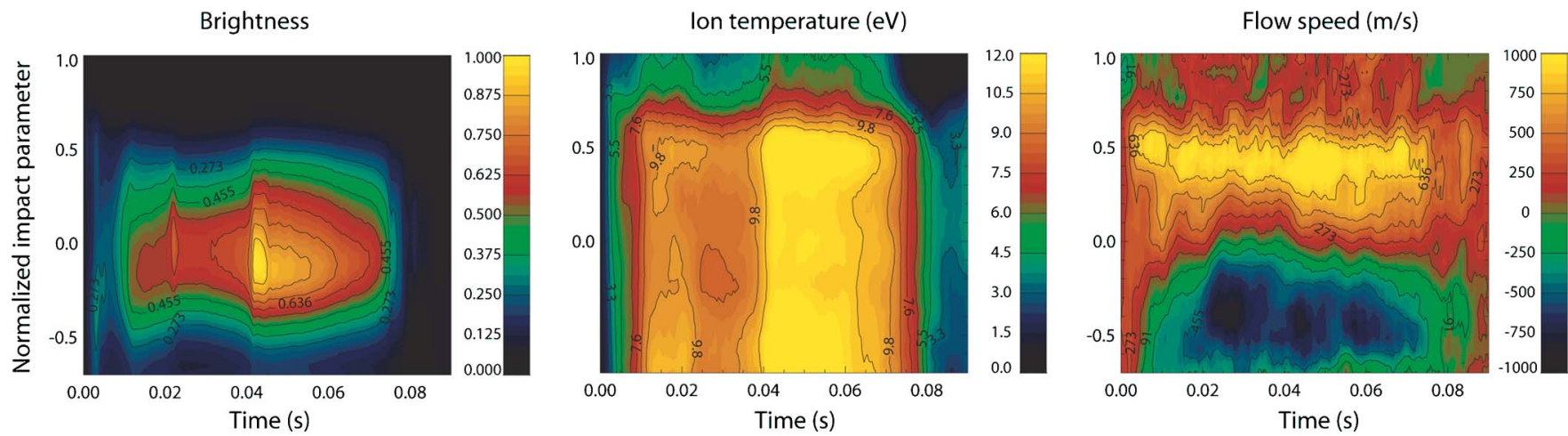

FIG. 3. Temporal evolution of brightness, ion temperature, and flow speed for an argon discharge exhibiting a transition from poor to improved confinement at $0.04 \mathrm{~s}$. At the transition the ion temperature increases from 10 to $12 \mathrm{eV}$, while there is little change in the plasma flow speed.

dual overlapping images, say, $I_{\text {top }}$ and $I_{\text {bottom. These images }}$ can be isolated using a split-field polarizer, constructed from adjoining, orthogonally oriented polarizers, that is located in the intermediate lens image plane (see Fig. 4). The separate, orthogonally polarized images are collimated by a second lens before traversing the remaining interferometer optics. In effect, the two images act as independent light sources that are angularly multiplexed through the interferometer and imaged onto the CCD detector. The relative optical delay of the dual images can be manipulated at the mask. For example, to produce quadrature images, a low order quarter wave plate can be cemented to one-half of the split-field polarizer. It is also possible to incorporate one or more color filters, which isolate spectral region(s) of interest at this first image.

The angularly separated images traverse a common birefringent delay plate before encountering the final polarizer, which is a second prism oriented to split the beams in a horizontal direction (orthogonal to the first prism). In conjunction with an imaging lens, four separate and independent images are formed on the detector. For each $I_{\text {top }}$ and $I_{\text {bottom, }}$, the left and right interferometric images $\left(S_{+}\right.$and $\left.S_{-}\right)$can be summed to give the total brightness image. The difference image normalized to the sum $\left(S_{+}-S_{-}\right) /\left(S_{+}+S_{-}\right)$gives the cophase component of the complex coherence [see Eq. (1)].

If a quarter wave delay is introduced into one of the images $I_{\text {top }}$ or $I_{\text {bottom }}$ at the split-polarizing mask, the resulting four images of the optical coherence will be in quadrature about a common fixed optical delay offset. The 4-quadrant image thereby gives a snapshot of the local interferogram phase and contrast without recourse to temporal multiplexing. This arrangement facilitates very fast imaging of simple spectral scenes using, for example, a framing streak camera. The quadrant polarization filter can be used with a gated intensified camera for imaging periodic phenomena, or repetitive pulse systems, when light levels are low. The system could also be used with imaging phototube arrays for continuous time monitoring of fluctuating quantities.

Both temporal and spatial multiplex coherence imaging systems have their merits and disadvantages. The simplest phase-stepped polarization interferometer discards $50 \%$ of the incident light at each of the polarizers and is not well suited for imaging transient events. While the quadrant imaging system utilizes all available light, overall throughput is not improved because only one-quarter of the detector area is used for any given interferometric image. While the temporal resolution of the static quadrant system is now limited only by the imaging technology and the available light flux, care must be exercised to ensure that the independent images are properly spatially registered prior to processing.

It is important to note that it is possible to perform Doppler coherence imaging of more complex multiplet structures provided only that the relative line intensities are fixed (e.g., Ref. 4). Where background emission is a problem, source modulation techniques (e.g., modulated diagnostic neutral beams) can be used to recover the Doppler information. ${ }^{10}$ Where this is not possible, a hybrid interferometer based on a combination of delay plates and interposed ferroelectric liquid crystal half wave plates, which switch in synchronism the camera frame rate, can be used to image the optical coherence of more complex scenes. Simpler variants of these

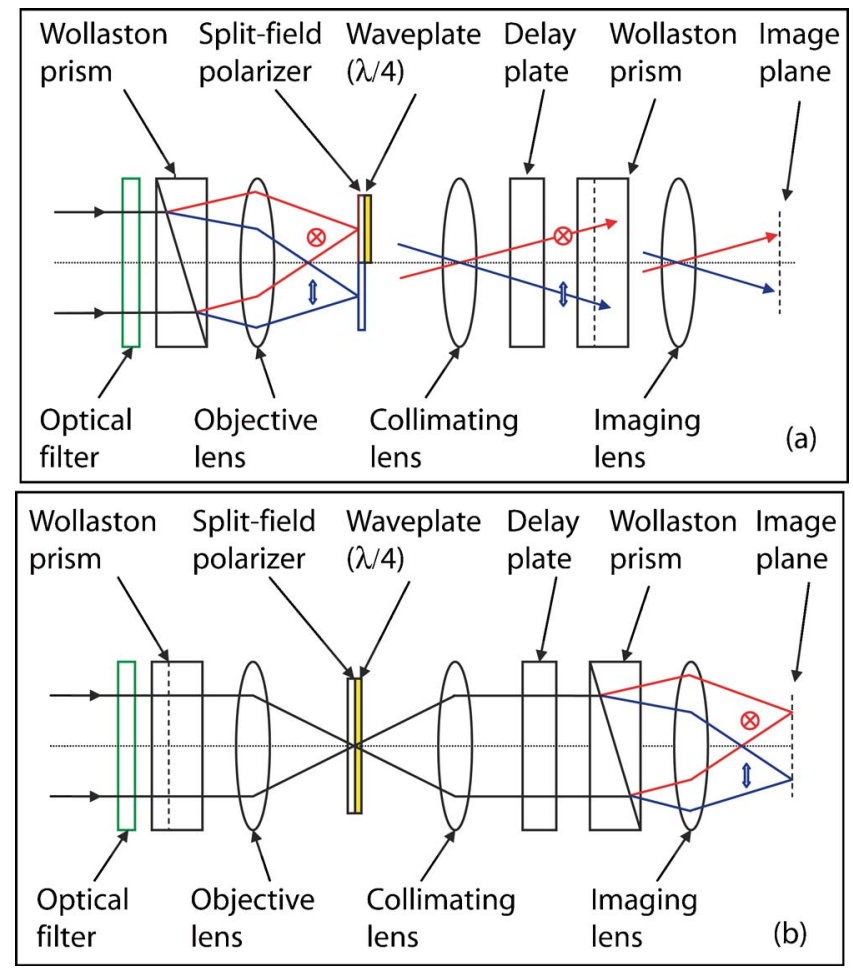

FIG. 4. Schematic view of optical layout for producing 4-quadrant coherence images of a quasimonochromatic source. See text for discussion. 
systems can also suffice in certain applications. For example, split-image systems have been proposed for use as spectral discriminators in laser Thomson scattering. ${ }^{5}$

\section{A. Optical design}

A first interference filter isolates the spectral region of interest. For the simple design shown here, the optical field of view (FOV) is then determined by the split angle of the first Wollaston lens. We have used a calcite Wollaston prism of clear aperture of $25 \mathrm{~mm}$ and full split angle of $5^{\circ}$. Together with an F-mount $35-80 \mathrm{~mm}$ zoom camera lens, the vertical separation of the orthogonal polarization images in the intermediate image plane is adjusted to give an appropriate image separation $(f \sim 45 \mathrm{~mm}$ gives $\sim 4 \mathrm{~mm})$. To increase the system's FOV we have used a negative lens of focal length $-400 \mathrm{~mm}$ located $\sim 900 \mathrm{~mm}$ from the plasma axis to produce a demagnified $(\times 0.3)$ virtual image $\sim 275 \mathrm{~mm}$ in front of the lens. In order to minimize vignetting effects the system's $f$ /number is set using the first lens, while retaining maximum aperture for subsequent lenses. For this system, we used the full available aperture $f / 4$ of the first zoom lens.

A second camera lens after the intermediate image plane collimates the radiation through the interferometer, where field-widened birefringent plates produce the desired optical path delay. For the measurements reported here we used high birefringence lithium niobate plates with total thickness of 30 and $40 \mathrm{~mm}$ corresponding to characteristic temperatures of 24 and $13 \mathrm{eV}$, respectively, for argon ion emission at $488 \mathrm{~nm}$.

The final Wollaston prism polarizes and splits the processed radiation in the horizontal direction. To retain the image aspect ratio, this prism also has a split angle of $5^{\circ}$. The $3 \mathrm{~mm}$ width of the patterned polarization mask is chosen to limit the horizontal extent of the intermediate image in order that the horizontally separated images formed at the final image plane do not overlap. A Roper Scientific Cascade 512B back-illuminated CCD camera with $512 \times 512$ pixels and array size of $8.2 \times 8.2 \mathrm{~mm}^{2}$ is used to image the four final images. The ratio of the final two lens focal lengths sets the magnification of the intermediate image onto the detector. Taking both lenses to have standard $50 \mathrm{~mm}$ focal length produces four images that fit comfortably within the CCD camera.

\section{B. Calibration procedure}

To obtain the instrument transmission efficiency $\eta_{I}$, fringe visibility $\zeta_{I}$, and phase $\phi_{I}$, it is necessary to image a spatially uniform, monochromatic light source that fills the instrument's field of view. A filtered spectral lamp or laser at wavelength close to that of the emission line of interest coupled to an integrating sphere can be used for this purpose. The interferometer response to a uniform monochromatic light source is then proportional to

$$
S=\eta_{I}\left(1+\zeta_{I} \zeta \cos \phi_{I}\right) \text {. }
$$

To determine the instrument function the optical phase must be scanned either by slightly tuning the light source wavelength (if this is possible) or by using a fixed wavelength source and scanning the optical path length either thermally

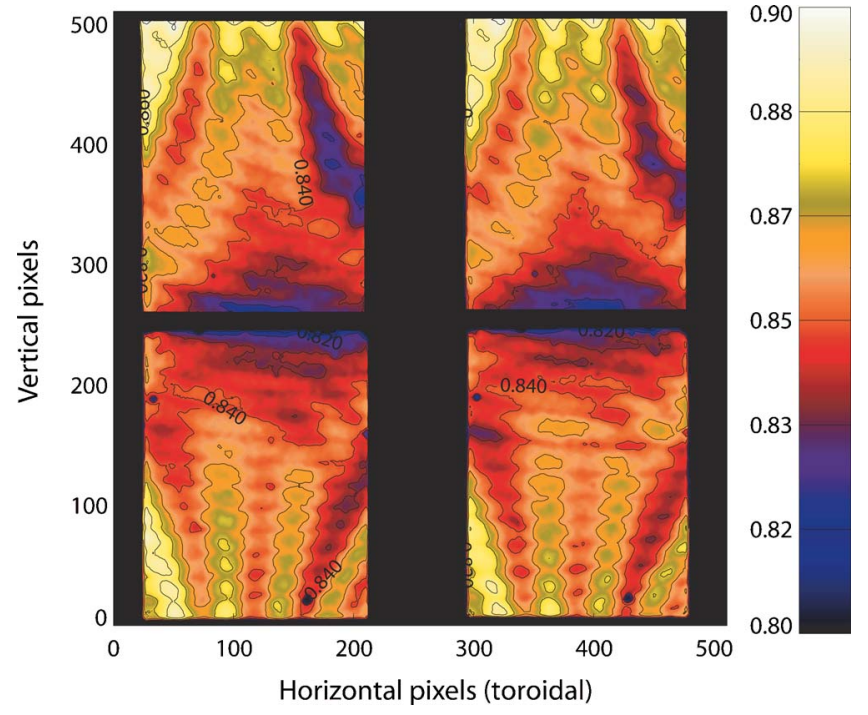

FIG. 5. Typical instrument contrast images for 4-quadrant system and fieldwidened delay plates $(2 \times 15 \mathrm{~mm}$ lithium niobate). Note the similarity of images within upper and lower pairs. Upper and lower pairs are different due to the quarter wave phase shifter. The residual contrast structure is mainly due to incomplete field-widening cancellation of the natural hyperbolic fringe patterns produced by the birefringent plates.

or electro-optically. We have chosen to step-scan the optical path length through approximately one wave period using a zero-net-delay electro-optic plate interposed between the birefringent delay plate and final Wollaston. The modulator is constructed from crossed, low-birefringence lithium tantalate (LT) plates each of thickness $10 \mathrm{~mm}$. Applying a voltage ramp step-synchronized with the camera frame rate produces a series of images that, in each pixel, captures a sinusoidal intensity pattern that can be numerically fitted to extract the required interferometric parameters. Although the LT optic introduces additional phase distortions which reduce slightly the overall fringe visibility, it is convenient to retain the modulator for routine calibration purposes. The calibration procedure produces images of transmission efficiency, instrument contrast, and phase for each of the four quadrants.

To verify the calibration, it is necessary to confirm that the instrument transmission and contrast images in each horizontally split pair are closely identical and that their phases differ by $\pi$ rad. To reduce noise propagation in the demodulation process, the calibration parameter images are subject to a five-point median filter and averaged for upper and lower quadrant pairs. The quantities $\left(\eta_{I}, \zeta_{I}, \phi_{I}\right)$ for the upper and lower image pairs are different due to the presence of the quarter wave plate at the image mask. Typical calibration image results are shown in Fig. 5.

The image acquisition, calibration, registration, and processing procedures are implemented using a LABVIEW application running on a personal computer (PC). The software controls and synchronizes an onboard data acquisition (DAQ) card, the camera, light shutter, and high voltage amplifier. Configuration and calibration information, as well as recorded images and signals, are stored in a self-contained MDSPlus database that is accessed by general recursive LABVIEW procedures. Before processing the independent images, it is necessary to subtract the background and spatially 

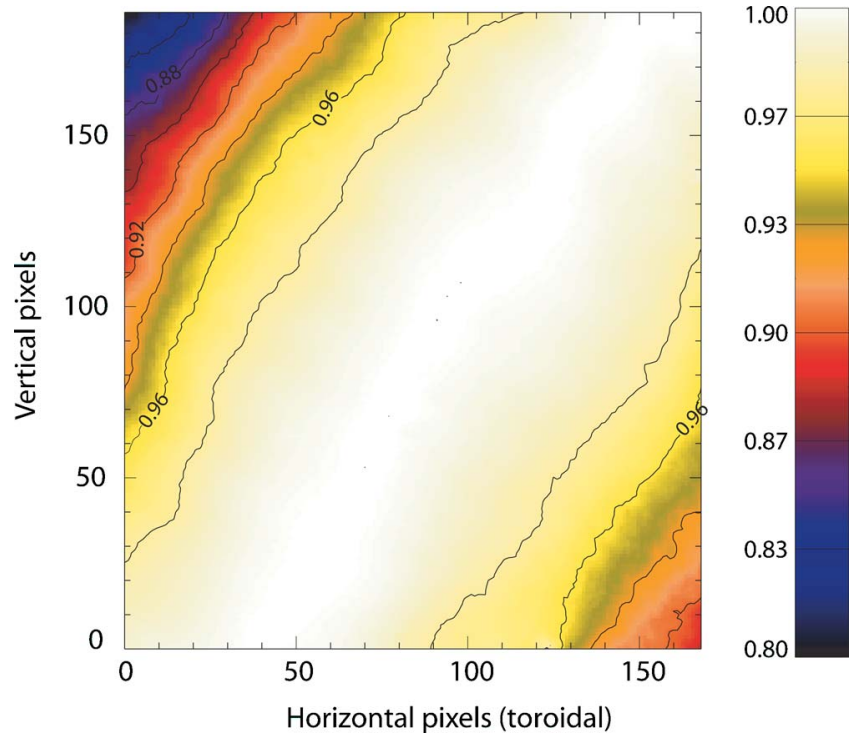

FIG. 6. Sine of the phase difference between the upper and lower calibration image pairs showing a high degree of quadrature across most of the image area.

register each of the four quadrants. This is implemented in LABVIEW using the add-on IMAQ-VISION utilities. In our case, the plasma-illuminated internal toroidal field coils provide a suitable image registration reference. In results presented below, we have not corrected for rotation or aberrations nor have we yet undertaken an analysis of the effects of registration errors.

\section{Image demodulation}

After correction for transmission efficiency, the signal in corresponding pixels in either of the upper or lower image pairs is proportional to

$$
\begin{aligned}
& S_{ \pm}=I_{0}\left[1 \pm \zeta_{I} \zeta \cos \left(\phi+\phi_{I}\right)\right] \\
& \equiv I_{0}\left(1 \pm \zeta_{I c} \zeta_{c} \mp \zeta_{I s} \zeta_{s}\right) \\
& \equiv I_{0}(1 \pm \gamma),
\end{aligned}
$$

where $\left(I_{0}, \zeta, \phi\right)$ are the emission line brightness, contrast, and phase [see Eqs. (8) and (10)]. To simplify notation we have introduced the instrument complex coherence $\zeta_{I C}+i \zeta_{I S}$ $\equiv \zeta_{I} \exp i \phi_{I}$ and plasma complex coherence $\zeta_{c}+i \zeta_{s}$ $\equiv \zeta \exp i \phi$, and defined the quantity $\gamma=\zeta_{I c} \zeta_{c}-\zeta_{I s} \zeta_{s}$. Using superscripts to denote top and bottom images, it is straightforward to extract the complex coherence of the plasma spectral line at the chosen offset delay as follows:

$$
\begin{aligned}
& \zeta_{s}=\left[\gamma^{(b)} \zeta_{I c}^{(t)}-\gamma^{(t)} \zeta_{I c}^{(b)}\right] / \Delta, \\
& \zeta_{c}=\left[\gamma^{(b)} \zeta_{I s}^{(t)}-\gamma^{(t)} \zeta_{I s}^{(b)}\right] / \Delta .
\end{aligned}
$$

The quantity $\Delta$ is related to the degree of orthogonality between the upper and lower image pairs and is given by

$$
\Delta=\zeta_{I s}^{(t)} \zeta_{I c}^{(b)}-\zeta_{I s}^{(b)} \zeta_{I c}^{(t)}=\zeta_{I}^{(b)} \zeta_{I}^{(t)} \sin \left[\phi_{I}^{(t)}-\phi_{I}^{(b)}\right] .
$$

Figure 6 shows an image of the quantity $\sin \left[\phi_{I}^{(t)}-\phi_{I}^{(b)}\right]$ that indicates a high degree of quadrature between the top and bottom image sets.

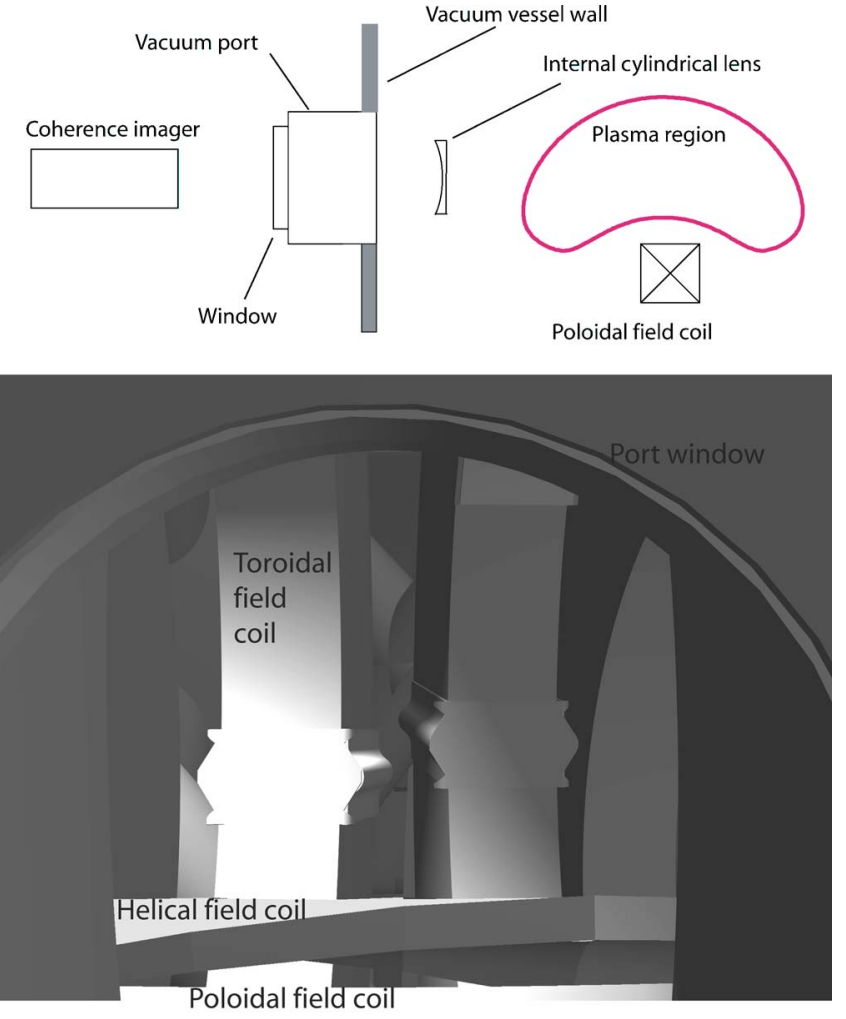

FIG. 7. Top: Schematic of plasma viewing arrangement. Below: AUTOCAD model of the camera view through the vacuum port window in the absence of a plasma. The vertical structures are the inside surfaces of the toroidal field coils (TFCs). The curved structure is the helical field coil (HFC) which sits atop the central poloidal field coil. The plasma sits above the HFC and is contained in the TFCs.

\section{STATIC COHERENCE IMAGING SYSTEM RESULTS}

To confirm the performance of the static system, measurements were performed on low field argon discharges under conditions similar to those reported above for the electrooptically modulated camera. The camera provided a maximum frame rate of $\sim 25 \mathrm{~Hz}$ at full resolution so that temporal resolution with the rf pulse was limited to two frames. Following the startup, the plasma conditions remain approximately steady so that the second of the two frames was used for the analysis.

A direct view along the major axis of the plasma was available for this work. An AUTOCAD model of the camera view of the plasma region looking through the vacuum tank port is shown in Fig. 7. Figure 8 shows a typical camera plasma snapshot. In each quadrant, illuminated by the plasma ion light, are two of the 36 internal toroidal field coils (TFCs) and their clamps. At the top of each image is the outline of the helical conductor. Each of the images is inverted so that the plasma actually resides above this conductor and is enclosed by the TFCs. Notice the presence of strong specular reflections from the leftmost TFC in the image.

The extracted brightness, temperature, and flow speed images for an argon discharge at $0.12 \mathrm{~T}$ in standard magnetic configuration and for a $30 \mathrm{~mm}$ lithium niobate delay plate $\left(T_{\mathrm{c}}=24 \mathrm{eV}\right)$ are shown in Fig. 9. A 5-pixel median filter has been applied to the temperature and flow images. Note that 


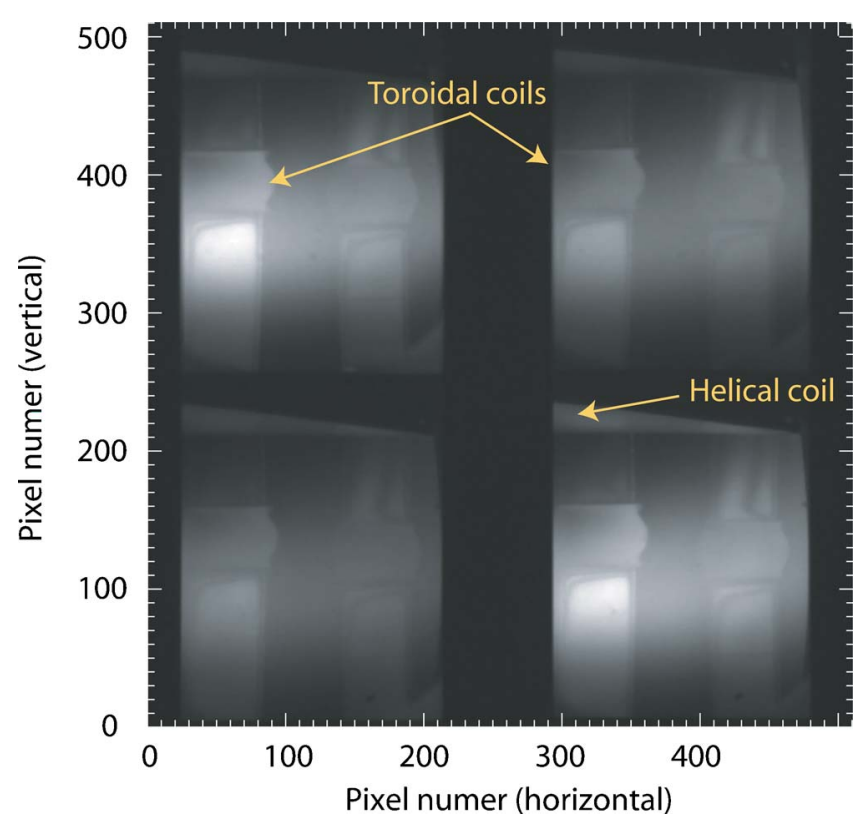

FIG. 8. Raw 4-quadrant $512 \times 512$ image. The individual images are inverted.

the static system reproduces all of the features shown in Fig. 3 . The ion temperature profile is substantially hollow, while the flow approximates rigid rotation. The absolute ion temperature is a little higher because of the larger magnetic field strength. The flow field is subjected to an arbitrary offset due to slow thermal drifts in the optical delay produced by the birefringent plate. We have shifted the flow so that it is approximately zero along the plasma centerline. Observe that the brightness, temperature, and flow images show little evidence of cross coupling.

Residual registration inaccuracies, which are evident in the flow and temperature images, are likely due to spherical distortions produced by the first simple negative lens, and which are not corrected by our simple shifting algorithm. Observe that the temperature image is artificially elevated in high flow regions, where light is also received by reflection from the coil surface. This is likely the result of the superposition of both blueshifted and redshifted light resulting in a decrease in fringe contrast.

It is instructive to study profiles of the measured parameters averaged over a region of interest that is 30 pixels wide and located midway between the TFCs. Profiles obtained for 30 and $40 \mathrm{~mm}$ delay plates $\left(T_{\mathrm{c}}=24\right.$ and $13 \mathrm{eV}$, respectively) are superimposed in Fig. 10. While the flow fields show good agreement, the deduced equivalent temperatures are quite inconsistent. As noted above, this effect, which increases towards the plasma edge, has been previously studied in some detail and has been satisfactorily explained in terms of a strong collisional coupling between the ion and background argon neutral fluids. ${ }^{9}$

This 2D imaging capability should be useful for observ-
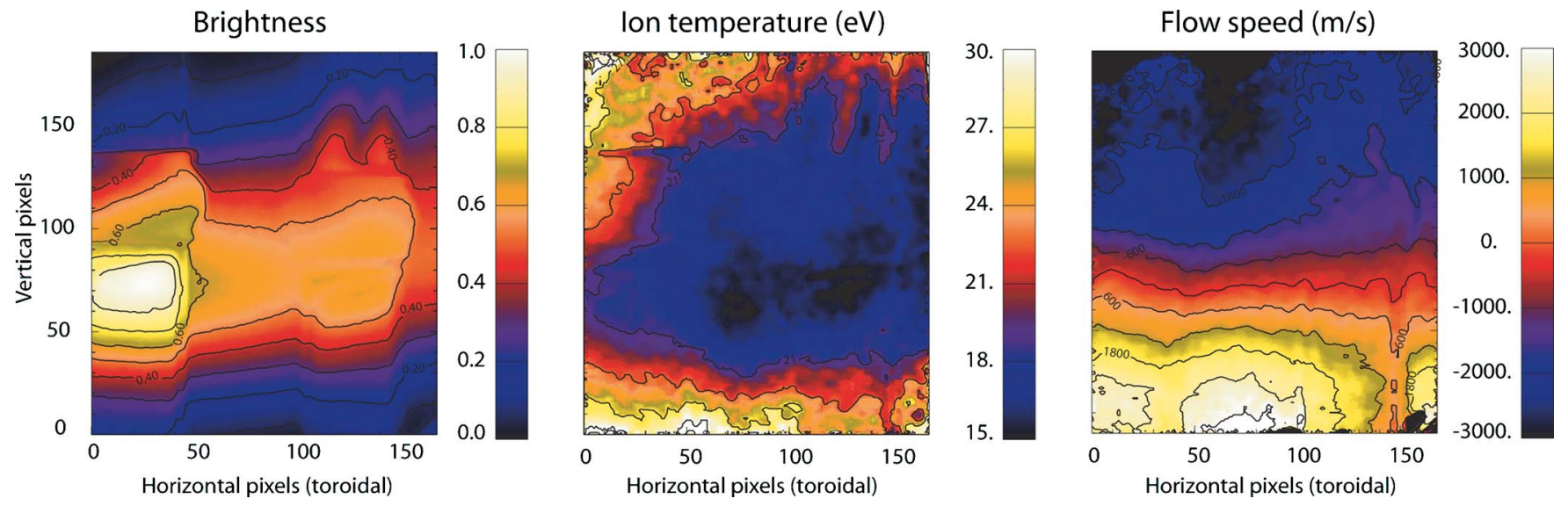

FIG. 9. Reduced 4-quadrant image showing average brightness, ion temperature, and flow fields. See text for discussion.

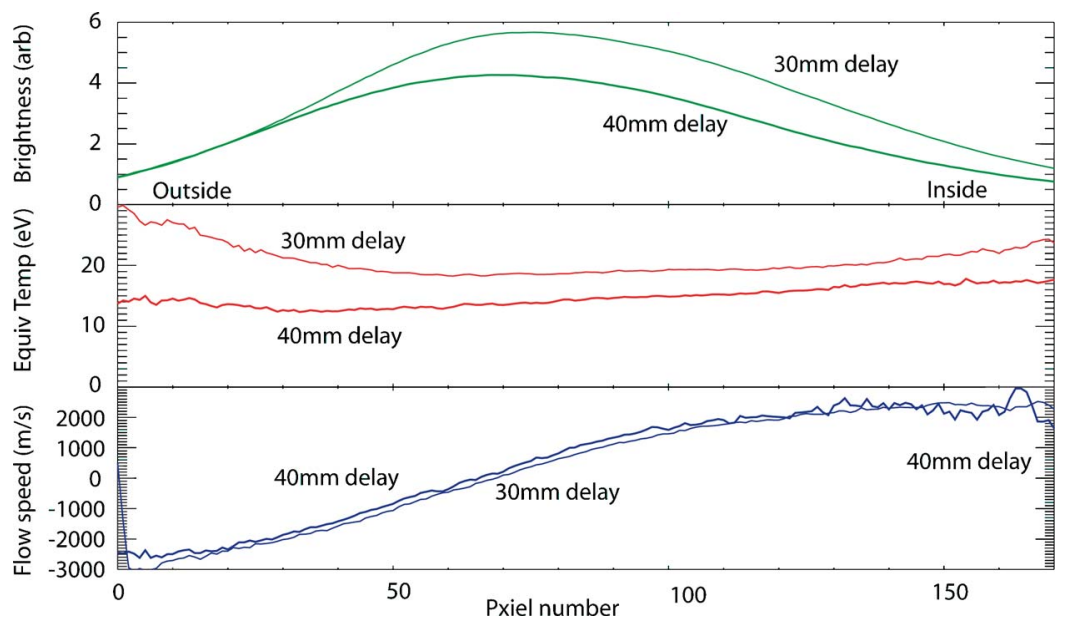

FIG. 10. Profiles of the brightness, equivalent temperature, and flow obtained using wave plates of delay 30 and $40 \mathrm{~mm}$. The disagreement between equivalent temperatures obtained using the different wave plates is explained by a significantly nonthermal ion velocity distribution function. The flow profiles are known to within a constant flow offset. 
ing asymmetric regions or structures in longer pulse plasma experiments. The 4-quadrant filter may also find applications when combined with gated, intensified CCD cameras. For example, by accumulating many frames synchronously with a rf heating source, it may be possible to image the temporal evolution of various species distributions within a rf wave period, leading to a better understanding of various wave absorption mechanisms. The method can also be adapted to other spectroscopic applications where the properties of the optical coherence can be simply related to the physical parameters that determine the spectral scene.

\section{ACKNOWLEDGMENT}

This work has been, in part, supported by the Australian Government Department of Education, Science and Training under the International Science Linkages program, Grant No. CG050061.

${ }^{1}$ J. Howard, C. Michael, F. Glass, and A. Cheetham, Rev. Sci. Instrum. 72, 888 (2001).

${ }^{2}$ J. Howard, Appl. Opt. 41, 197 (2002).

${ }^{3}$ J. Howard, C. Michael, F. Glass, and A. Danielsson, Plasma Phys. Controlled Fusion 45, 1143 (2003).

${ }^{4}$ J. Chung, R. Konig, T. Klinger, and J. Howard, Plasma Phys. Controlled Fusion 47, 919 (2005).

${ }^{5} \mathrm{~J}$. Howard (unpublished).

${ }^{6}$ C. Michael, J. Howard, and B. D. Blackwell, Rev. Sci. Instrum. 72, 1034 (2001).

${ }^{7}$ F. Glass, J. Howard, and B. Blackwell, IEEE Trans. Plasma Sci. 33, 472 (2005).

${ }^{8}$ W. Steel, Interferometry (Cambridge University Press, Cambridge, 1967).

${ }^{9}$ C. Michael, J. Howard, and B. Blackwell, Phys. Plasmas 11, 4008 (2004).

${ }^{10}$ J. Howard, L. Carraro, M. E. Puiatti, F. Sattin, P. Scarin, M. Valisa, B. Zaniol, R. Koing, and J. Chung, Rev. Sci. Instrum. 74, 2060 (2003). 